\title{
EphrinA1/EphA2 Promotes Epithelial Hyperpermeability Involving in Lipopolysaccharide-induced Intestinal Barrier Dysfunction
}

\author{
Yuhua Chen, ${ }^{1}$ Lei Zhang, ${ }^{1 *}$ Yongbo Zhang, ${ }^{1,2}$ Tao Bai, ${ }^{1}$ Jun Song, ${ }^{1}$ Wei Qian, ${ }^{1}$ and Xiaohua Hou ${ }^{1 *}$ \\ ${ }^{1}$ Division of Gastroenterology, Union Hospital, Tongji Medical College, Huazhong University of Science and Technology, Wuhan, China; and \\ ${ }^{2}$ Department of Gastrointestinal Surgery, Shandong Provincial Qianfoshan Hospital, Shandong University, Jinan, China
}

\begin{abstract}
Background/Aims
Lipopolysaccharide (LPS) is the key factor inducing mucosal and systemic inflammation in various intestinal and parenteral diseases, which could initially disrupt the epithelial barrier function. EphrinA1/ephA2 is speculated to increase the epithelial permeability for its "repulsive interaction" between adjacent cells. This study aim to investigate the role of ephrinA1/ephA2 in LPS-induced epithelial hyperpermeability.
\end{abstract}

\section{Methods}

In vivo model challenged with oral LPS in C57BL/6 mice and in vitro model exposed to LPS in Caco2 monolayer were established. The barrier function was assessed including expression of tight junction proteins (occludin and claudin-1), transepithelial electrical resistance, and permeability to macromolecules (fluorescein isothiocyanate-labeled fluorescent dextran 4 kDa [FD4]). Moreover, the expression and phosphorylation of ephrinA1/ephA2 were quantified, and its roles in the process of epithelial barrier disruption were confirmed via stimulating ephA2 with ephrinA1-Fc chimera (ephrinA1-Fc) and inactivating ephA2 with ephA2-Fc chimera (ephA2-Fc), or ephA2 monoclonal antibody (ephA2-mab), as well as inhibiting extracellular signal-regulated kinase 1/2 (ERK1/2) with PD98059.

\section{Results}

LPS induced significant barrier dysfunction with dismissed occludin and claudin-1 expression, reduced transepithelial electrical resistance and increased FD4 permeability, accompanied by upregulated ephrinA1/ephA2 pathway and phosphorylation of ephA2 receptor. Furthermore, ephA2-Fc, and ephA2-mab ameliorated LPS-induced epithelial hyperpermeability, which was also inhibited by PD98059. Additionally, ephrinA1-Fc led to apparent epithelial leakage in Caco2 monolayer by promoting the phosphorylation of ERK1/2, which could be obviously blocked by ephA2-mab and PD98059.

\section{Conclusion}

EphrinA1/ephA2 promotes epithelial hyperpermeability with an ERK1/2-dependent pathway, which involves in LPS-induced intestinal barrier dysfunction.

(J Neurogastroenterol Motil 2020;26:397-409)

\section{Key Words}

Lipopolysaccharides; Receptor, EphA2; Receptor, Fc; Tight junction proteins

Received: April 26, 2019 Revised: April 1, 2020 Accepted: May 6, 2020

(3) This is an Open Access article distributed under the terms of the Creative Commons Attribution Non-Commercial License (http://creativecommons. org/licenses/by-nc/4.0) which permits unrestricted non-commercial use, distribution, and reproduction in any medium, provided the original work is properly cited.

*Correspondence: Lei Zhang and Xiaohua Hou are equally responsible for this study.

Lei Zhang, MD, PhD

Division of Gastroenterology, Union Hospital, Tongji Medical College, Huazhong University of Science and Technology, Wuhan 430022, China Tel: +86-02785726113, Fax: +86-02785726113, E-mail: zhanglei2017@hust.edu.cn

Xiaohua Hou, MD, PhD

Division of Gastroenterology, Union Hospital, Tongji Medical College, Huazhong University of Science and Technology, Wuhan 430022, China Tel: +86-02785726057, Fax: +86-02785726057, E-mail: houxh@hust.edu.cn 
inflammation in condition of bacterial endotoxin challenge.

\section{Introduction}

Gut-derived bacterial lipopolysaccharide (LPS) plays an essential role in inducing intestinal and systemic inflammatory responses, ${ }^{1,2}$ which has been implicated as a pathogenic factor in various intestinal and parenteral diseases, such as inflammatory bowel disease, necrotizing enterocolitis, alcoholic liver disease, acute pancreatitis, and critical illnesses. ${ }^{3-5}$ Moreover, physiological factors such as psychological stress, high-fat diet and ageing can also lead to elevated LPS levels in the gut and in the circulation. ${ }^{6,7}$ Overall, increased intestinal epithelial permeability should be the common initiating mechanism in these processes.

LPS may directly act on the epithelial monolayer and destroy the intestinal barrier function. ${ }^{8}$ Normally, the concentration of LPS in the gut lumen has been reported to be about $1.8 \mu \mathrm{g} / \mathrm{mL}$ as detected in the rat distal ileum. ${ }^{9,10}$ Elevated LPS in the lumen ascribed to intestinal dysbiosis may trigger the dysfunction of the epithelial barrier. It has been demonstrated that LPS exceeding $2 \mu \mathrm{g} / \mathrm{mL}$ was enough to increase the permeability of $\mathrm{Caco} 2$ monolayers with dismissed intercellular tight junctions. ${ }^{911}$ However, the specific mechanisms under barrier damage and tight junction disrupt by LPS exposure has not been well established.

EphrinA1 and ephA2 membrane receptors may mediate "repulsive interaction" between adjacent cells, which belong to the largest receptor tyrosine kinase family. ${ }^{12}$ In particular, ephA2 represents a subtype in the erythroprotein-producing human hepatocellular carcinoma (eph) receptors, and ephrinA1 is a ligand for ephA2, which represents a subtype in eph family receptor-interacting proteins (ephrins). ${ }^{13,14}$ Several studies have focused on the complex role of ephrinA1/ephA2 signaling in vascular endothelial barrier function and inflammatory response. ${ }^{15,16}$ Particularly, activating ephA2 receptor by ephrinA 1 could increase the permeability of brain microvascular endothelium and lung alveolar epithelium. ${ }^{13,16,17}$ LPS exposure increased the activity of ephrinA1/ephA2 signaling, which destroyed the pulmonary epithelial barrier integrity with declined E-cadherin accumulation and tight junctions. ${ }^{13,15}$ It has been shown that ephrinA1 and ephA2 are highly expressed in intestinal epithelium. ${ }^{18}$ Therefore, ephrinA1/ephA2 is speculated to be involved in the intestinal epithelial hyperpermeability upon LPS exposure. Whereas, the underlying mechanisms remain to be clarified.

In this study, we aim to investigate the role of ephrinA1/ephA2 in LPS-induced epithelial hyperpermeability and its molecular mechanism in vivo and in vitro. The results may further provide potential targets for modulating the epithelial barrier function and

\section{Materials and Methods}

\section{Animals and Colitis Models}

Male C57BL/6 mice (aged 8 weeks, weighed 18-22 g; Experimental Animal Center, Tongii Medical College, HUST, Wuhan, China) were used. All mice were housed in specific pathogen-free conditions with 12-hour/12-hour light/dark cycle, and free access to food and water at $23^{\circ} \mathrm{C}$. The colitis models were induced via intragastric administration of LPS (from Escherichia coli O111:B4) for 3 consecutive days, $1 \mathrm{mg} / \mathrm{kg}$ or $10 \mathrm{mg} / \mathrm{kg}$ body weight per day respectively; while the control mice were intragastrically infused with equivalent distilled water. Finally, all mice were anesthetized and sacrificed, the colonic mucosa were rapidly stripped and used for epithelial permeability tests as detailed below: the colonic tissue were collected and fixed in 4\% paraformaldehyde for histopathological and immunohistochemical analysis, or fixed in $2 \%$ glutaraldehyde for transmission electron microscopical analysis as standard procedures. Additionally, the remaining colonic samples were stored at $-80^{\circ} \mathrm{C}$ for quantitative mRNA and protein tests. All experiment procedures were performed in accordance with the ethical guidelines of the Animal Management Rules of the Chinese Ministry of Health (Document No. 55, 2001), and approved by the Animal Care and Use Committee, Union Hospital, Tongji Medical College, HUST, China (Approval ID [2016] No. S153).

\section{Ussing Chamber and Mucosal-to-Serosal Permeability Test}

The mucosal tissues were mounted on the center of U-type chambers filled with $37^{\circ} \mathrm{C}$ oxygenated Krebs' solution, which was installed on the Ussing Chamber System (World Precision Instruments, Sarasota, FL, USA). After a 20-minute equilibration period, the transepithelial electrical resistance (TEER) was recorded via an automatic voltage clamp model. Then, $1 \mathrm{mg} / \mathrm{mL}$ fluorescein isothiocyanate-labeled fluorescent dextran $4 \mathrm{kDa}$ (FD4) or 40 $\mathrm{kDa}$ (FD40), which represented the paracellular or transcellular macromolecular permeability respectively, was added to the mucosal side of the U-type chambers, and sampled in the serosal side at 30-minute intervals over a 2-hour period. The FD4 and FD40 intensity was detected by a Fluorescence Microplate Reader (Bio Tek Instruments, Winooski, VT, USA). The mucosal-to-serosal permeability was calculated with increased FD4 or FD40 transmission within 2 hours. 


\section{Caco2 Cell Monolayer Culture and Interventions}

Caco2 cell line was obtained from American Type Culture Collection (ATCC; Manassas, VA, USA), which has been widely used as a model of the intestinal epithelial barrier. As previously described, the cells were cultured in Iscove's Modified Dulbecco's Medium (IMDM) with $50 \mathrm{U} / \mathrm{mL}$ penicillin, $50 \mathrm{U} / \mathrm{mL}$ streptomycin, $2 \mathrm{mM}$ glutamine, and $10 \%$ fetal bovine serum, in an incubator with $95 \% \mathrm{O}_{2}+5 \% \mathrm{CO}_{2}$ at $37^{\circ} \mathrm{C}$. The culture medium was changed every 2 days. Particularly, cells $\left(2 \times 10^{5} /\right.$ well $)$ were seeded and grew in 6-well plate for 2 weeks before interventions, which could form a functional epithelial monolayer barrier. The $E$. coli O111:B4 LPS (1, 10, or $100 \mu \mathrm{g} / \mathrm{mL}$ respectively) was added into the culture medium and incubated for 24 hours to establish an epithelial barrier injury model. Accordingly, $2.5 \mu \mathrm{g} / \mathrm{mL}$ ephA2Fc (the ephA2 receptor inhibitor), $2.0 \mu \mathrm{g} / \mathrm{mL}$ ephA2-mab (the ephA2 monoclonal antibody), or $10 \mu \mathrm{g} / \mathrm{mL}$ PD98059 (the extracellular signal-regulated kinase 1/2 [ERK1/2] inhibitor) was posttreated as necessary to investigate the role of ephrinA1/ephA2 and mitogen-activated protein kinase (MAPK)/ERK in this process.

\section{Transepithelial Electrical Resistance and Permeability Assay}

Caco2 cells were plated on the transwell filters with $0.4 \mu \mathrm{m}$ pore size (Corning, New York, USA) to form an epithelial monolayer. The TEER was measured via an $\mathrm{EVOM}^{2}$ volt-ohmmeter (World Precision Instruments), and calculated as $\Omega \cdot \mathrm{cm}^{2}$. The TEER reached $500 \Omega \cdot \mathrm{cm}^{2}$ indicated formation of functional tight junction between Caco2 monolayers after 2-week culturing. Then, it could be used for further barrier studies. The transepithelial permeability was assessed by means of apical-to-basolateral FD4 transmission. Briefly, $0.5 \mathrm{mg} / \mathrm{mL}$ FD4 was added in the up-chamber (apical side) for 30 minutes, then sampled at the down-chamber (basolateral side) and detected by a Fluorescence Microplate Reader (Bio Tek Instruments). The transepithelial permeability was presented as concentration of FD4 in the basolateral chamber.

\section{Western Blotting and Immunoprecipitation Analysis}

Total protein of colonic tissues or Caco2 cells were extracted via radioimmunoprecipitation assay (RIPA) buffer containing phosphatase and protease inhibitor cocktail. For immunoblotting analysis, lysates were separated on $10 \%$ sodium dodecyl sulphatepolyacrylamide gel electrophoresis (SDS-PAGE) gels with constant voltage (100 V, 90 minutes), and transferred to polyvinylidene fluoride (PVDF) membranes with constant current (300 mA,
90 minutes) in ice-water baths. Membranes were subsequently blocked in 5\% (weight/volume) bovine serum albumine, incubated with specific primary antibodies (occludin, claudin-1, ephrinA1, or ephA2; Abcam, Cambridge, MA, USA) and horseradish peroxidase-conjugated second antibodies, finally the protein bands were developed by a Super Signal West Pico Substrate (Pierce, Rockford, IL, USA) and quantified on the Fluor Chem FC3 system (ProteinSimple, San Jose, CA, USA). For immunoprecipitation assays, protein lysates were pre-cleared with agarose-protein A/G (Beyotime, Beijing, China), and then incubated with antiphosphotyrosine antibody, clone 4G10 (pre-incubated with agroseprotein $A / G$ ), centrifugated and the deposition was collected (immunoprecipitating complex). Subsequently, the complex was used for electrophoretic analysis by immunoblotting with anti-ephA2 antibodies as described above.

\section{Immunofluorescence Staining Analysis}

For paraffin section (fixed with $4 \%$ paraformaldehyde), routine dewaxing and hydration and then use for staining was performed. For the Caco 2 monolayer cell slide, fixation in $4 \%$ paraformaldehyde for 15 minutes was used for staining. Firstly, the paraffin sections or cell slides were blocked with $10 \%$ donkey serum for 1 hour at room temperature. Next, the preparations were incubated with primary antibodies (occludin, claudin-1, ephrinA1, or ephA2; Abcam) overnight at $4^{\circ} \mathrm{C}$, fully washed and combined with Alexa Fluor 488 secondary antibodies (Invitrogen, Carlsbad, CA, USA) for 60 minutes at room temperature. Finally, the nuclei were stained with 4',6-diamidino-2-phenylindole (DAPI, $1 \mu \mathrm{g} / \mathrm{mL}$; Beyotime). The fluorescent imaging was viewed on a confocal laser scanning microscope (Nikon, Tokyo, Japan), and analyzed using NIS Elements Viewer Software (Nikon).

\section{Scratch Wound Assay}

Scratch wound assay was performed as the standard procedure. Briefly, Caco2 cells were seeded on 6-well plates and starved for 12 hours in IMDM medium with $1 \%$ fetal bovine serum. Then, a scratch wound was made by creating a linear cell-free region using a pipette tip, washed off the detached cells and achieved a smooth scratch edge. The plates were transferred to the incubator. Cell migration was photographed and recorded by measuring the gap $(\mu \mathrm{m})$ between the edges of the scratch under an inverted microscope (Olympus, Tokyo, Japan) at 0 hour and 24 hours.

\section{Statistical Methods}

Data were presented as mean \pm SEM. The SPSS 20.0 (IBM 

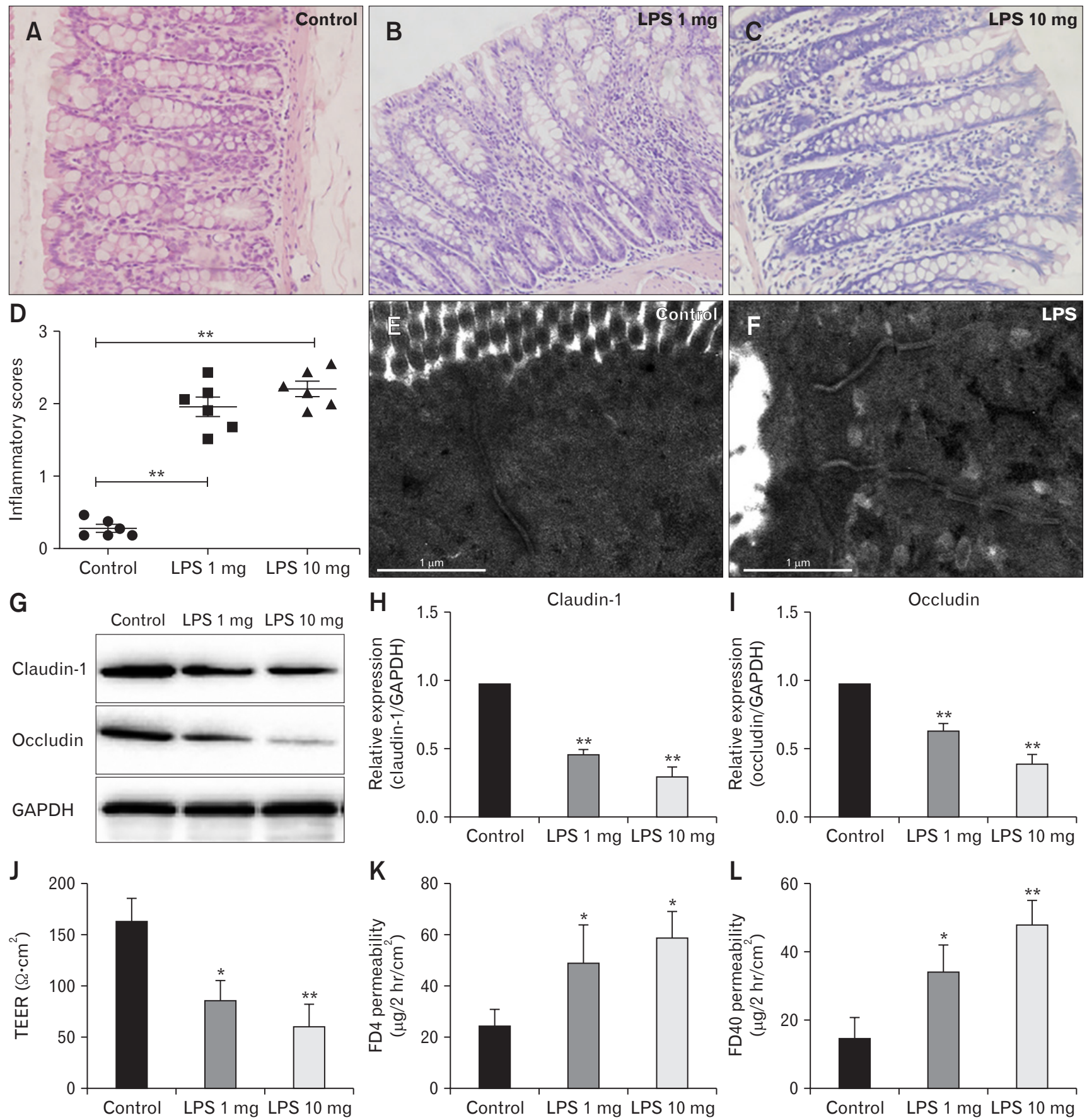

Figure 1. Lipopolysaccharide-induced apparent colonic epithelial hyperpermeability and inflammation in vivo. (A-D) Oral LPS (1 mg/kg and $10 \mathrm{mg} / \mathrm{kg}$ body weight) induced significant mucosal inflammation in the colon observed under H\&E staining, with increased histological scores. Original magnification: $\times 200$. (E, F) Disruption of ultrastructural epithelial tight junction in the colon exposure to LPS. (G-I) LPS treatment reduced the expression of tight junction proteins, claudin-1 and occludin. (J-L) LPS challenge destroyed the barrier function in the colon with declined transepithelial electrical resistance (TEER), and both the paracellular fluorescein isothiocyanate (FITC)-labeled fluorescent dextran 4 $\mathrm{kDa}$ (FD4) permeability and the transcellular FITC-labeled fluorescent dextran $40 \mathrm{kDa}$ (FD40) permeability. $\mathrm{n}=6,{ }^{*} P<0.05,{ }^{* *} P<0.01$ vs controls. GAPDH, glyceraldehyde-3-phosphate dehydrogenase. 
Corp, Armonk, NY, USA) was utilized for statistical analyses. Statistical significance between groups was analyzed by one-way ANOVA, followed with the least significant difference test or Dunnett's T3 test accordingly. It was considered statistically significant when $P<0.05$.

\section{Results}

\section{Lipopolysaccharide-induced Apparent Colonic} Epithelial Hyperpermeability and Upregulated the Level of Epithelial EphrinA1/EphA2 In Vivo

LPS-treated mice showed a significant mucosal inflammation in the colon with apparent inflammatory cell infiltration (Fig. 1AC) and increased histological scores (Fig. 1D). The ultrastructure of the epithelial tight junction was disrupted in colon exposure to LPS (Fig. 1E and 1F), with decreased expression of claudin-1 and occludin, the tight junction proteins (Fig. 1G-I). The barrier function was therefore destroyed under LPS challenge in the colon with declined TEER (Fig. 1J), and both the paracellular FD4 permeability and the transcellular FD40 permeability (Fig. 1K and 1L). It might be more influenced by LPS exposure in which received a higher dose treatment. Moreover, LPS induced obvious increase of ephrinA1 and ephA2 expression on the colonic epithelium (Fig. 2A and $2 \mathrm{~B}$ ), and further confirmed by the protein quantification (Fig. 2C-E).

\section{Lipopolysaccharide Disrupted the Barrier Function of Caco2 Monolayer Cells and Increased the Expression of EphrinA1/EphA2 In Vitro}

LPS destroyed the barrier function of $\mathrm{Caco} 2$ monolayer with dismissed intracellular tight junctions (Fig. 3A and 3B), and downregulated the expression of occludin and claudin-1 in a concentration-dependent way (Fig. 3C and 3D). Also, the expression of ephrinA1 was increased by LPS exposure concentrationdependently, but not for the expression of ephA2 receptor (Fig. 4A and $4 \mathrm{~B}$ ). However, phosphorylated level of ephA2 is significantly elevated in the LPS-treated monolayer (Fig. 4C and 4D), which indicated increased activation of ephA2 induced by LPS.

\section{Blocking of EphA2 Ameliorated the Barrier Dysfunction Induced by Lipopolysaccharide in Caco2 Monolayer}

EphA2 receptor blocking with specific ephA2 inhibitor, both
A

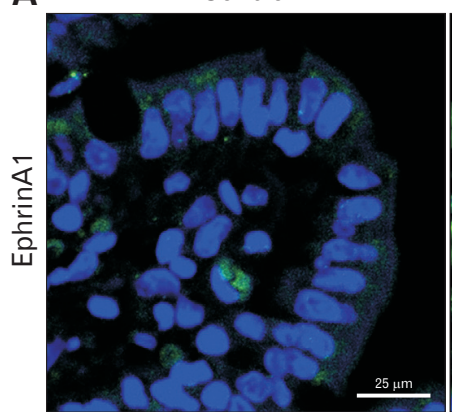

C

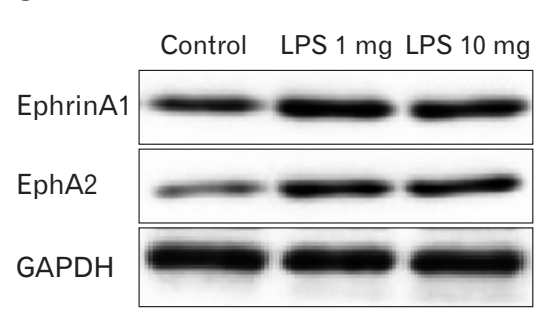

LPS

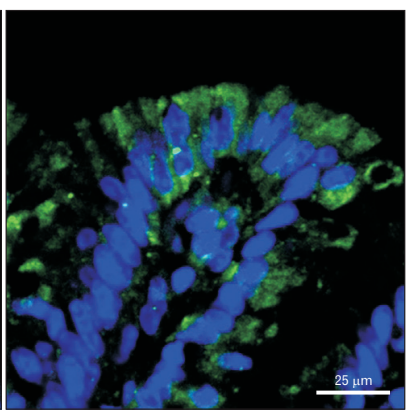

D

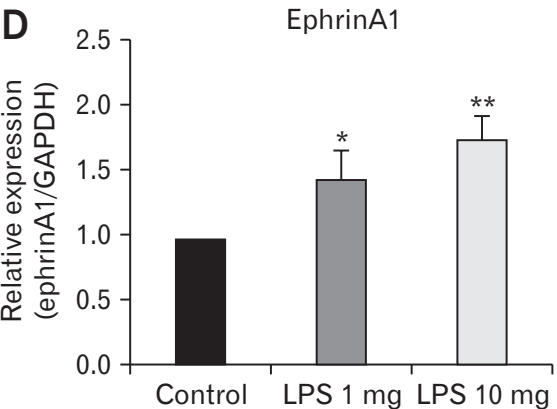

Control

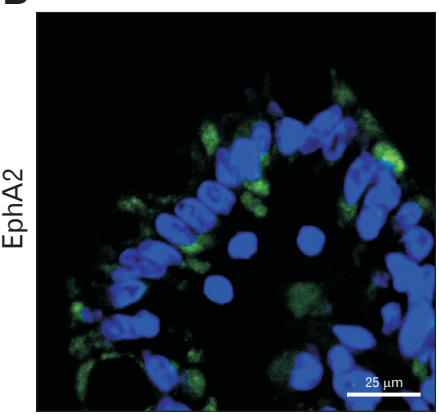

E

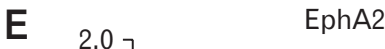

LPS

EphA2
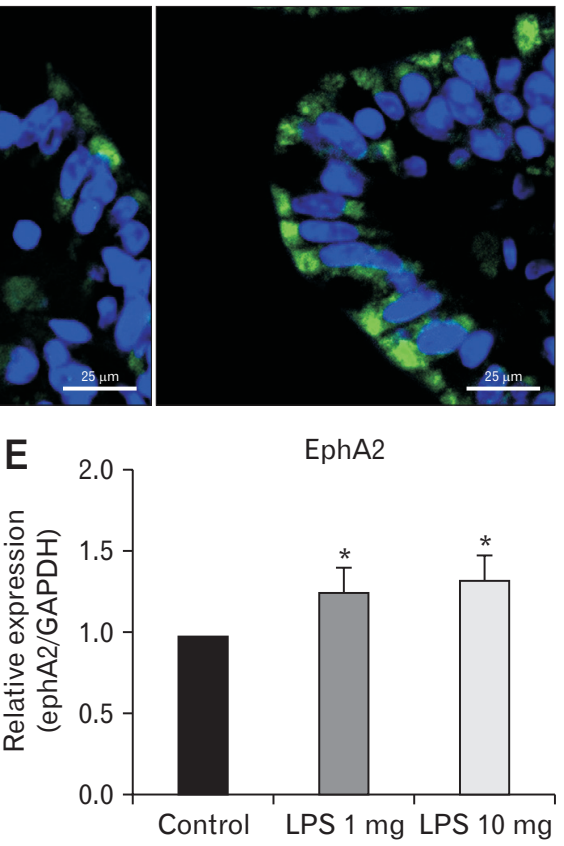

Figure 2. Lipopolysaccharide increased the levels of epithelial ephrinA1/ephA2 in the colon in vivo. (A, B) LPS induced obvious increase of ephrinA1 and ephA2 expression on the colonic epithelium observed with immunofluorescence staining. Green represent ephrinA1 or ephA2; blue represent nuclei (4',6-diamidino-2-phenylindole [DAPI]). (C-E) LPS stimulated increased expression of ephrinA1/ephA2 protein by immunoblotting quantification. $\mathrm{n}=6,{ }^{*} \mathrm{P}<0.05,{ }^{* *} \mathrm{P}<0.01$ vs controls. GAPDH, glyceraldehyde-3-phosphate dehydrogenase. 
A

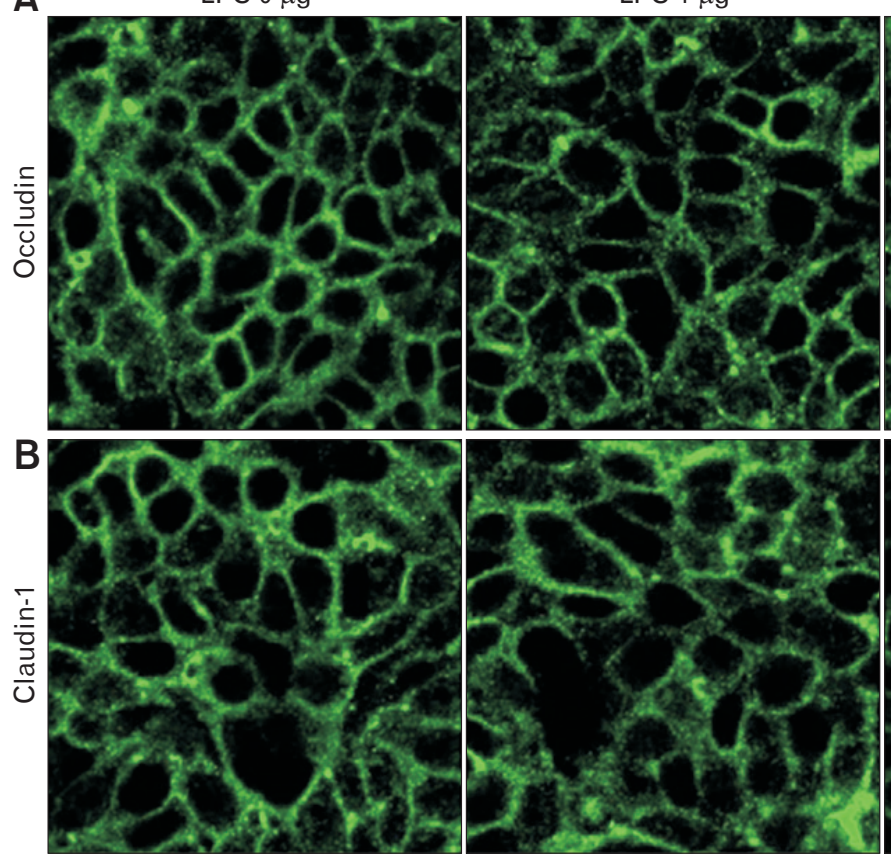

C
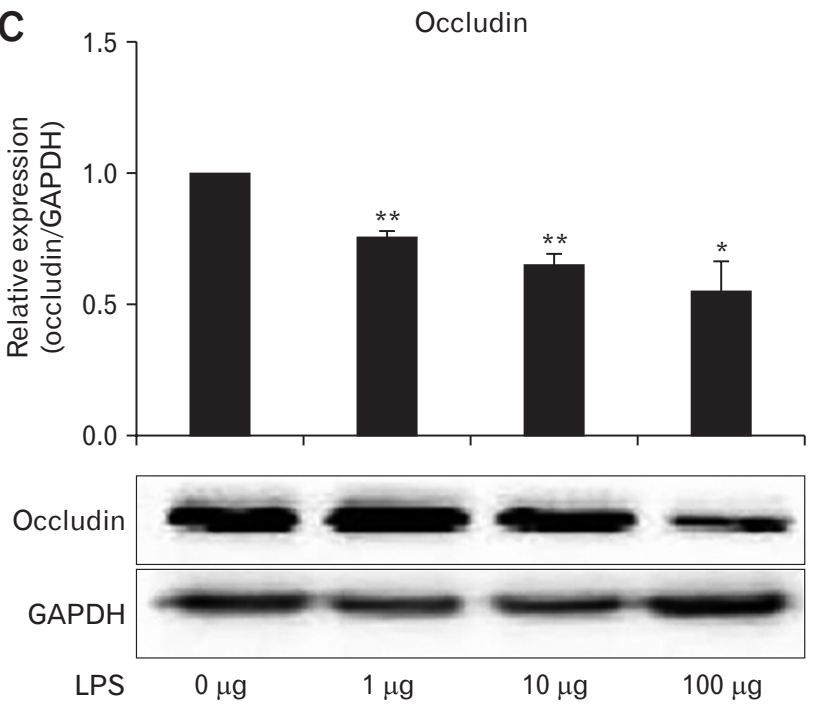

LPS $10 \mu \mathrm{g}$
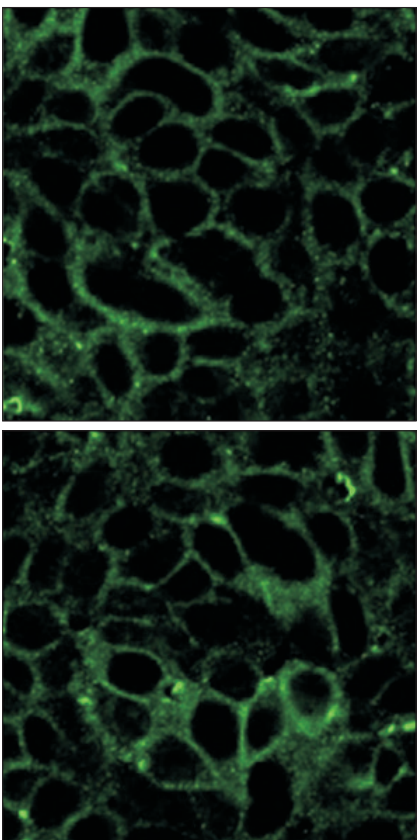

D
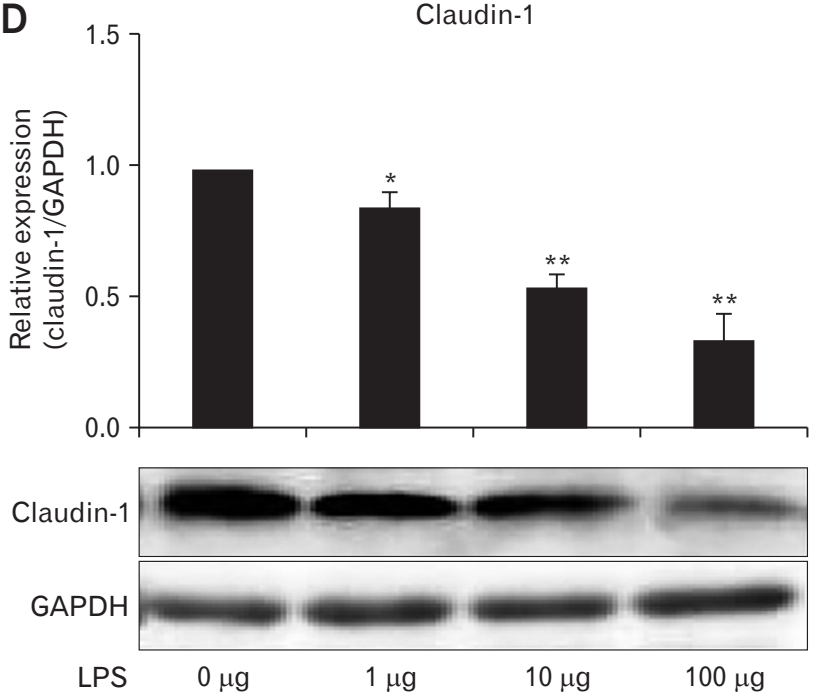

Figure 3. Lipopolysaccharide disrupted the barrier function of $\mathrm{Caco} 2$ monolayer in vitro. (A, B) LPS destroyed the barrier function of Caco2 monolayer with dismissed intracellular tight junctions in a concentration-dependent way. The typical immunofluorescent images of occludin and claudin-1. Original magnification: $\times 400$. (C, D) LPS concentration-dependently downregulated the expression of occludin and claudin-1 quantified by western blotting. $\mathrm{n}=8-10,{ }^{\star} P<0.05,{ }^{*} P<0.01$ vs vehicle (LPS $0 \mu \mathrm{g}$ ). GAPDH, glyceraldehyde-3-phosphate dehydrogenase.

the ephA2-Fc chimera (ephA2-Fc) and the ephA2 monoclonal antibody (ephA2-mab), ameliorated the LPS-induced barrier dysfunction by repairing the intracellular tight junctions (Fig. 5A) with increased expression of occludin and claudin-1 (Fig. 5B and 5C). LPS induced marked decrease of TEER and increase of FD4 permeability, which may be improved by ephA2-Fc and ephA2-mab (Fig. 5D and 5E), suggesting the activation of ephA2 played a role in this LPS-mediated barrier dysfunction of Caco2 monolayer.

EphrinA1/EphA2 Activation Promoted the Epithelial Hyperpermeability Involving the Phosphorylation of Extracellular Signal-regulated Kinase 1/2 Cascade

EphA2 receptor activation by specific ephA2 agonist, the ephrinA1-Fc chimera (ephrinA1-Fc), induced significant disruption 

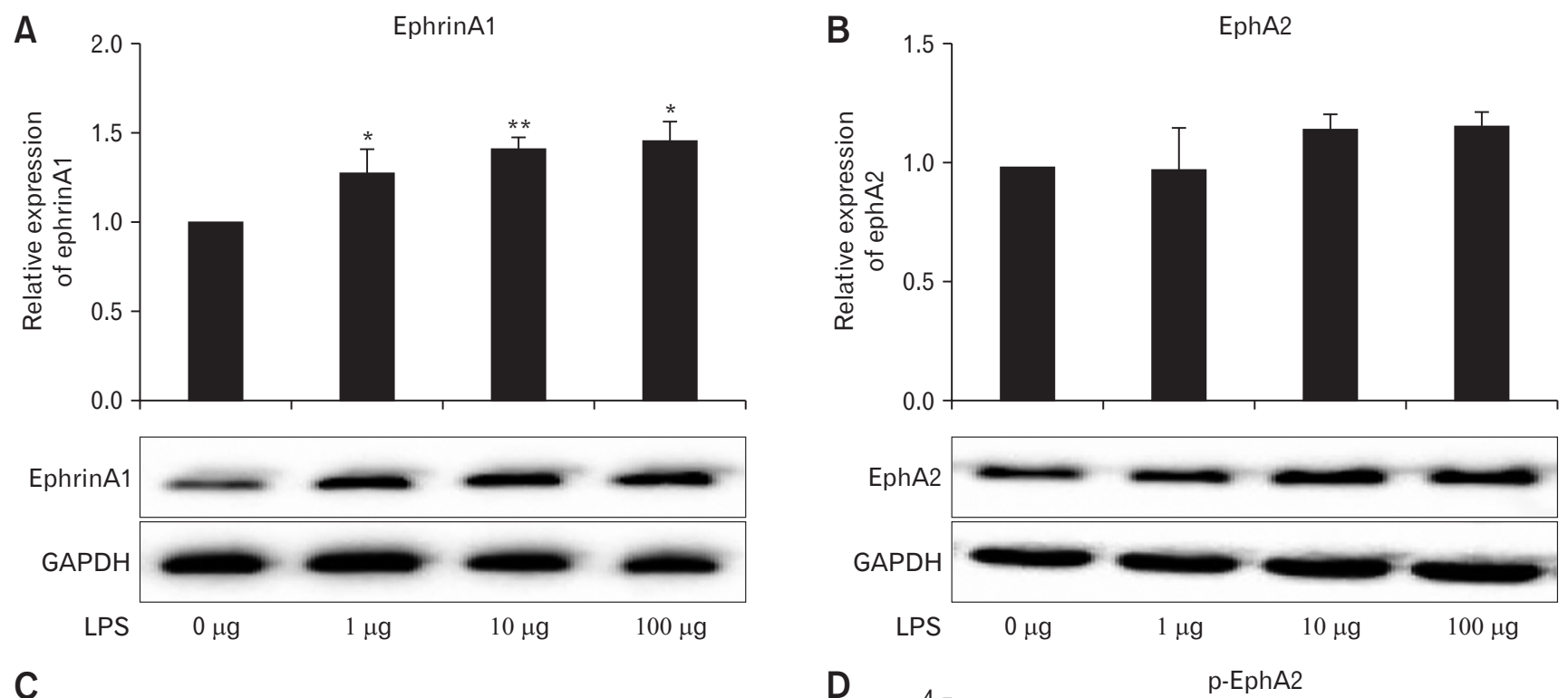

C

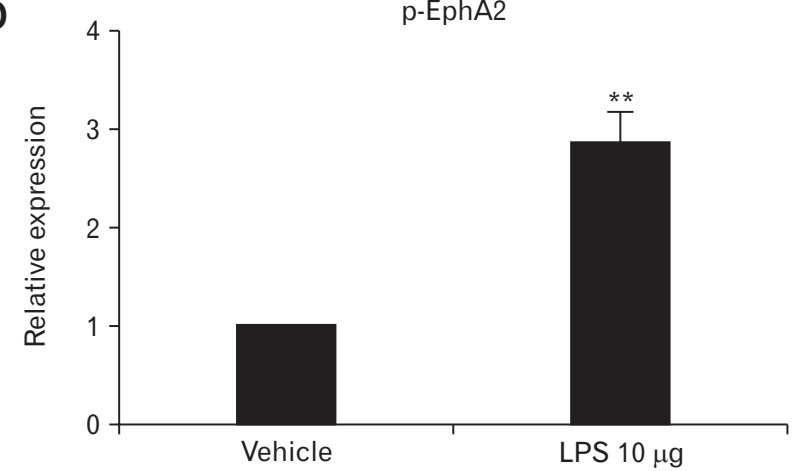

Figure 4. Lipopolysaccharide promoted the expression and phosphorylation of ephrinA1/ephA2 in Caco2 monolayer in vitro. (A, B) LPS exposure increased the expression of ephrinA1, but not for the expression of ephA2 receptor. (C, D) The phosphorylated level of ephA2 (p-ephA2) is significantly elevated in the LPS-treated monolayer, which indicated increased activation of ephA2 induced by LPS. $n=8-10,{ }^{*} P<0.05,{ }^{*} P<$ 0.01 vs vehicle (LPS $0 \mu \mathrm{g}$ ). GAPDH, glyceraldehyde-3-phosphate dehydrogenase; IP, immunoprecipitation; IB, immunoblotting; 4G10, antiphosphotyrosine antibody (clone 4G10).

of barrier dysfunction with dismissed intracellular tight junctions (Fig. 6A) and decreased expression of occludin and claudin-1 (Fig. 6B and 6C) in $\mathrm{Caco} 2$ monolayer. It may be markedly ameliorated by ephA2-mab, as well as PD98059, the specific ERK1/2 inhibitor (Fig. 6A-C). Also, in the functional aspect, ephrinA1-Fc stimulated the apparent decline of TEER and increase of FD4 permeation in the Caco2 monolayer, and alleviated by ephA2-mab and PD98059 (Fig. $6 \mathrm{D}$ and $6 \mathrm{E}$ ). Furthermore, it showed that LPS exposure led to significant increase of phosphorylation of ERK1/2 (p-ERK1/2), but not change of the total ERK1/2, which could be obviously inhibited by ephA2-Fc and ephA2-mab (Fig. 7A and 7B). Similarly, ephrinA1-Fc mimicked the effects of LPS with increased p-ERK1/2, while relieved by ephA2-mab and PD98059 (Fig. 7C and 7D). It suggested the ERK1/2 pathway in ephrinA1/ephA2-mediated and
LPS-induced barrier disruption in Caco2 monolayers.

\section{EphrinA1/EphA2-Extracellular Signal-regulated Kinase 1/2 Facilitated the Cell Migration in $\mathrm{Caco} 2$ Epithelium}

The scratch wound assay showed that activation of ephA2 receptor with ephrinA1-Fc accelerated the cell migration and wound repair, which indicated weakened intercellular tight junctions. Particularly, ERK1/2 inhibition with PD98059 prevented the cell migration mediated by ephrinA1-Fc in Caco2 monolayers (Fig. 8A and $8 \mathrm{~B})$. 

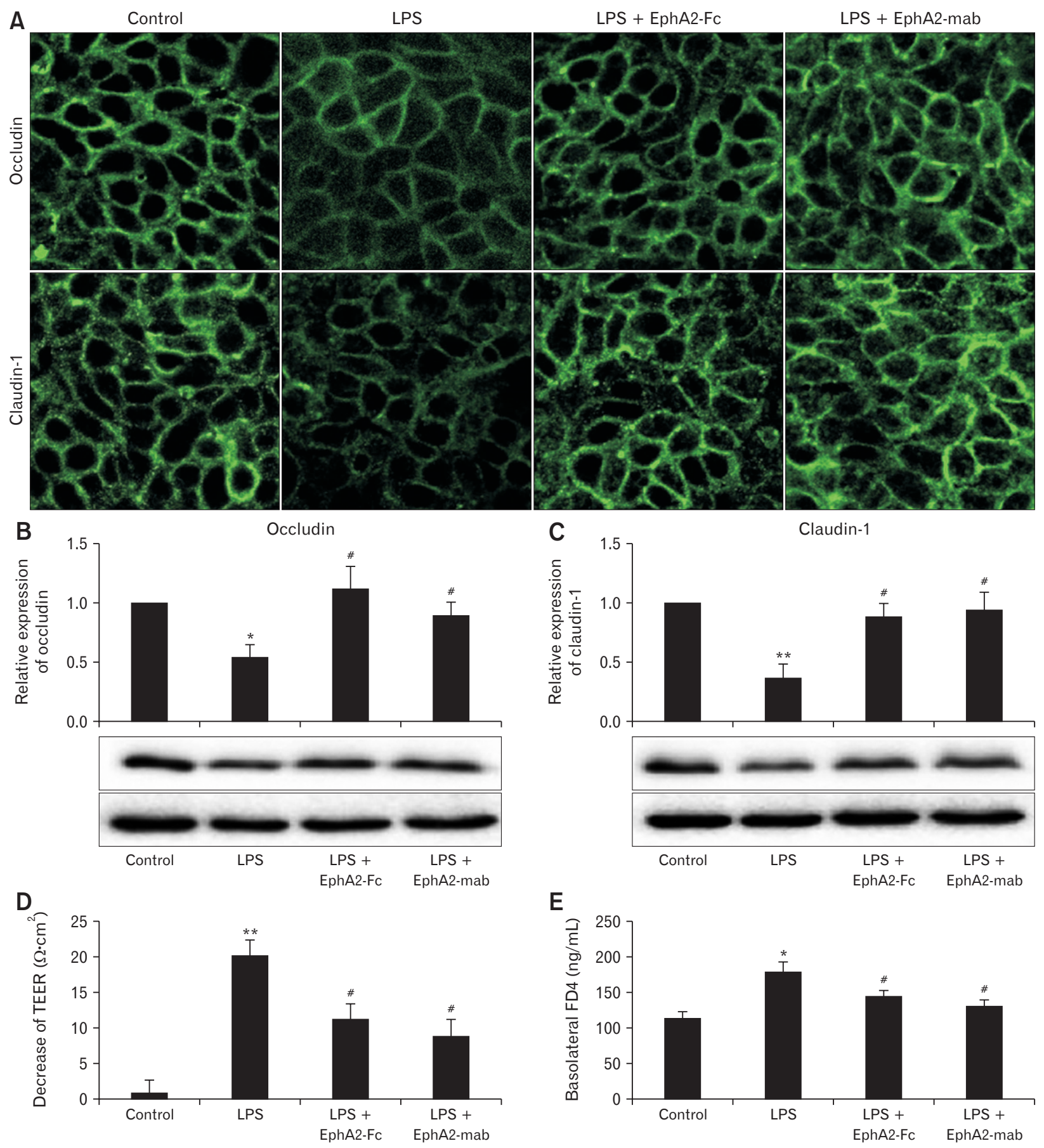

Figure 5. Blocking of ephA2 ameliorated the barrier dysfunction induced by lipopolysaccharide (LPS, $10 \mu \mathrm{g} / \mathrm{mL}$ ) in Caco2 monolayer. (A) EphA2 receptor blocking by ephA2-Fc chimera (ephA2-Fc, $2.5 \mu \mathrm{g} / \mathrm{mL}$ ) and ephA2 monoclonal antibody (ephA2-mab, $2.0 \mu \mathrm{g} / \mathrm{mL}$ ) ameliorated the LPS-induced barrier dysfunction by repairing the intracellular tight junctions. The typical immunofluorescent images of occludin and claudin-1. Original magnification: $\times 400$. (B, C) LPS-induced obvious decline of occludin and claudin-1 expression were relieved by ephA2-Fc and ephA2-mab. (D, E) LPS induced marked decrease of transepithelial electrical resistance (TEER) and increase of fluorescein isothiocyanatelabeled fluorescent dextran $4 \mathrm{kDa}$ (FD4) permeability, which may be improved by ephA2-Fc and ephA2-mab. $\mathrm{n}=8-10,{ }^{*} P<0.05,{ }^{* *} P<0.01$ vs controls; ${ }^{\#} \mathrm{P}<0.05$ vs LPS. GAPDH, glyceraldehyde-3-phosphate dehydrogenase. 

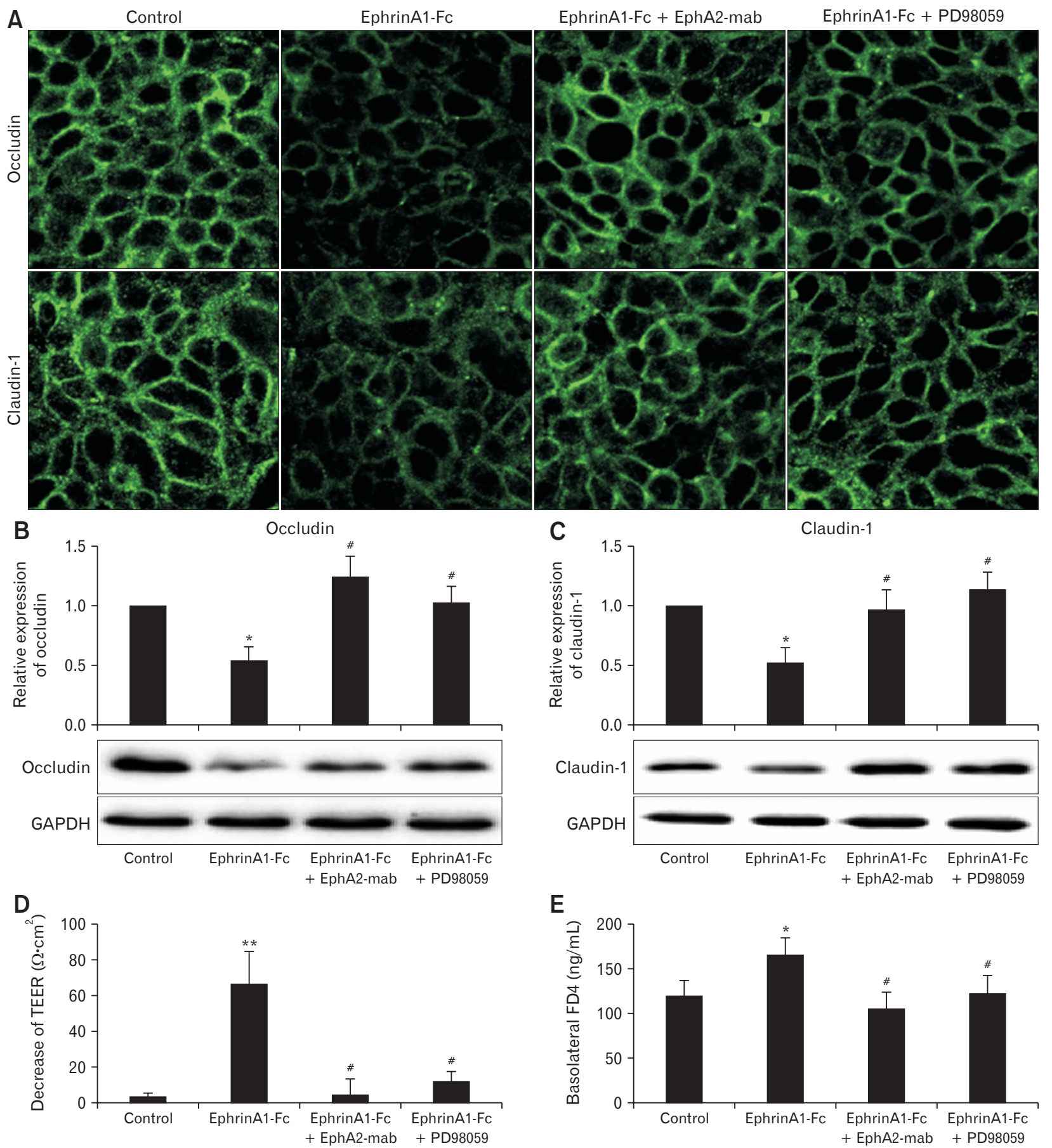

Figure 6. EphrinA1/ephA2 activation promoted the epithelial hyperpermeability in Caco2 monolayer. (A) EphA2 receptor activation by ephrinA1Fc chimera (ephrinA1- Fc, $2.5 \mu \mathrm{g} / \mathrm{mL}$ ) induced significant disruption of barrier dysfunction with dismissed intracellular tight junctions, while ameliorated by ephA2 monoclonal antibody (ephA2-mab, $2.0 \mu \mathrm{g} / \mathrm{mL}$ ) and extracellular signal-regulated kinase 1/2 inhibitor (PD98059; $10 \mu \mathrm{g} /$ $\mathrm{mL})$. The typical immunofluorescent images of occludin and claudin-1. Original magnification: $\times 400$. (B, C) EphrinA1-Fc reduced the expression of occludin and claudin-1, and increased in those treated with ephA2-mab and PD98059. (D, E) EphrinA1-Fc stimulated apparent decline of transepithelial electrical resistance (TEER) and increase of fluorescein isothiocyanate-labeled fluorescent dextran $4 \mathrm{kDa}(\mathrm{FD} 4)$ permeation in Caco2 monolayer, also alleviated by ephA2-mab and PD98059. $\mathrm{n}=8-10,{ }^{*} P<0.05,{ }^{* *} P<0.01$ vs controls; ${ }^{*} P<0.05$ vs LPS. GAPDH, glyceraldehyde-3-phosphate dehydrogenase. 
A

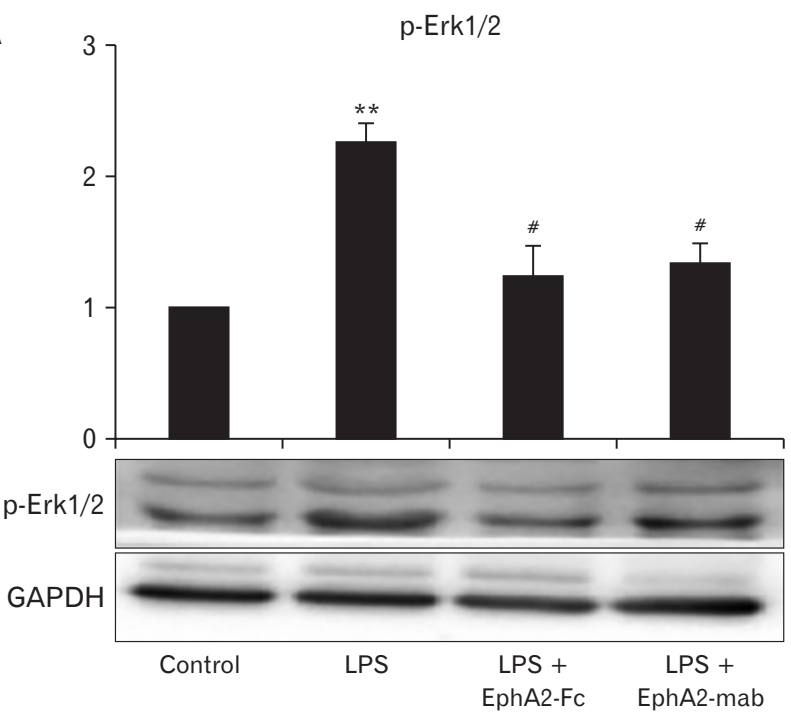

C

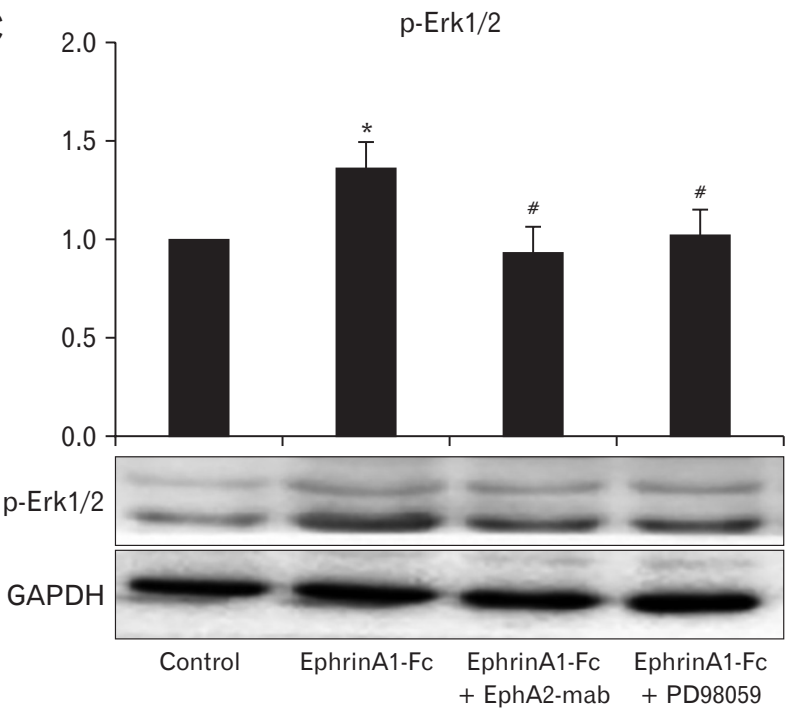

B

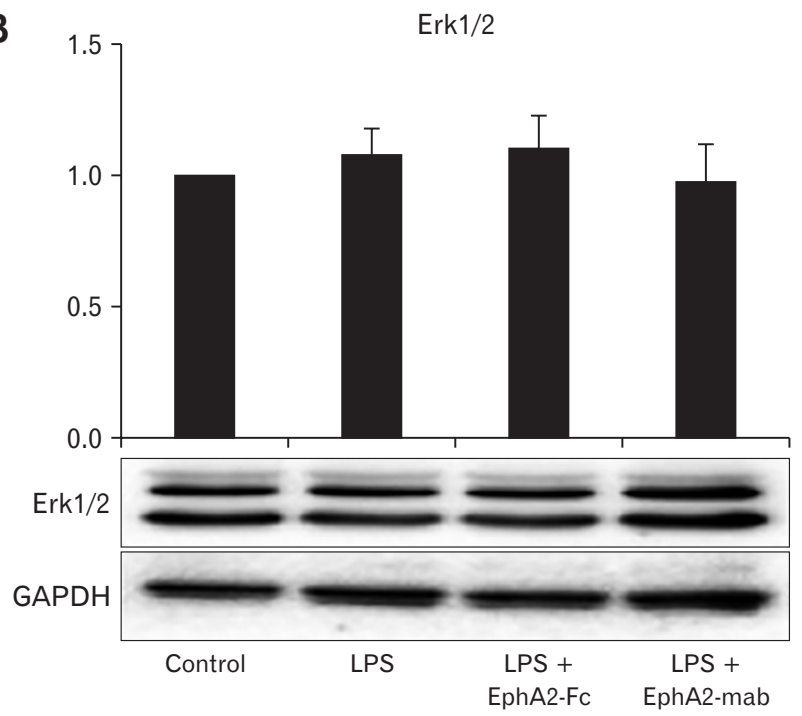

Erk1/2

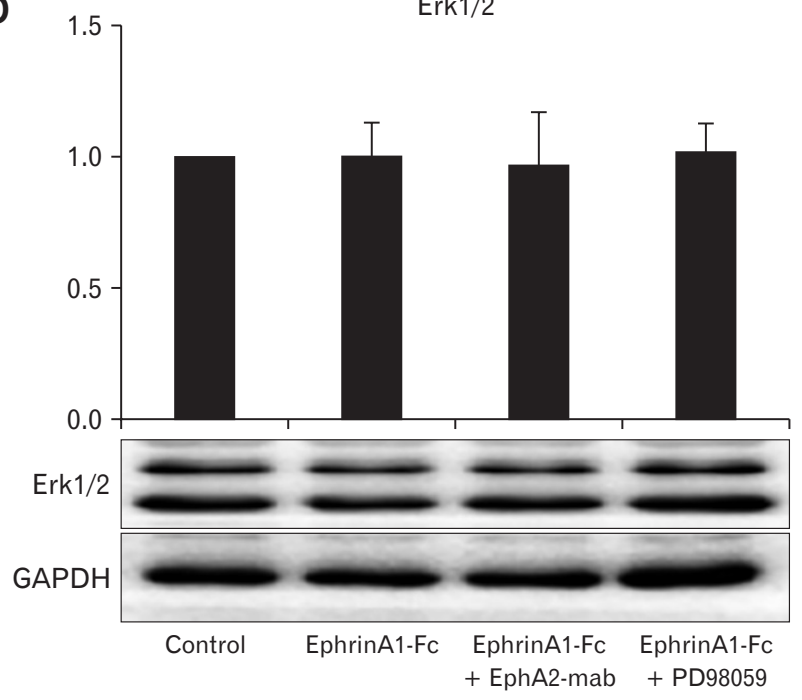

Figure 7. EphrinA1/ephA2 mediated epithelial hyperpermeability involving the phosphorylation of extracellular signal-regulated kinase $1 / 2$ (ERK1/2) signaling. (A, B) LPS exposure led to significant increase of phosphorylation of ERK1/2 (p-ERK1/2), but not change of the total ERK1/2, which could be obviously inhibited by ephA2-Fc chimera (ephA2-Fc) and ephA2 monoclonal antibody (ephA2-mab). (C, D) EphrinA1-Fc chimera (ephrinA1-Fc) treatment increased levels of p-ERK1/2, while relieved by ephA2-mab and ERK1/2 inhibitor (PD98059) in Caco2 monolayers. $\mathrm{n}=8-10,{ }^{*} \mathrm{P}<0.05,{ }^{*} \mathrm{P}<0.01$ vs controls; ${ }^{*} \mathrm{P}<0.05$ vs LPS. GAPDH, glyceraldehyde-3-phosphate dehydrogenase.

\section{Discussion}

Recent studies have reported that the altered intestinal permeability was regarded as a key factor in the development of digestive and non-digestive diseases. ${ }^{19}$ Therefore, cellular and molecular therapeutic targets in those diseases should be focused on the recovery of intestinal barrier function. As our results found, up-regulated ephrinA1/ephA2 expression and phosphorylation were associated with LPS-induced intestinal epithelial barrier destruction. Furthermore, the epithelial hyperpermeability could be mimicked by ephrinA1-Fc exposure, while relieved by ephA2-Fc or ephA2mab treatment, as well as ERK1/2 inhibition. The ephA2 receptor and ephrinA1 ligand were located on the colonic epithelium and upregulated in the inflammatory gut of LPS-treated mice as we shown. This is consistent with previously described that increased ephrinA1/ephA2 expression in dextran sulfate sodium-induced colitis. ${ }^{20}$ Others also demonstrated that ephA2 signaling was crucial 

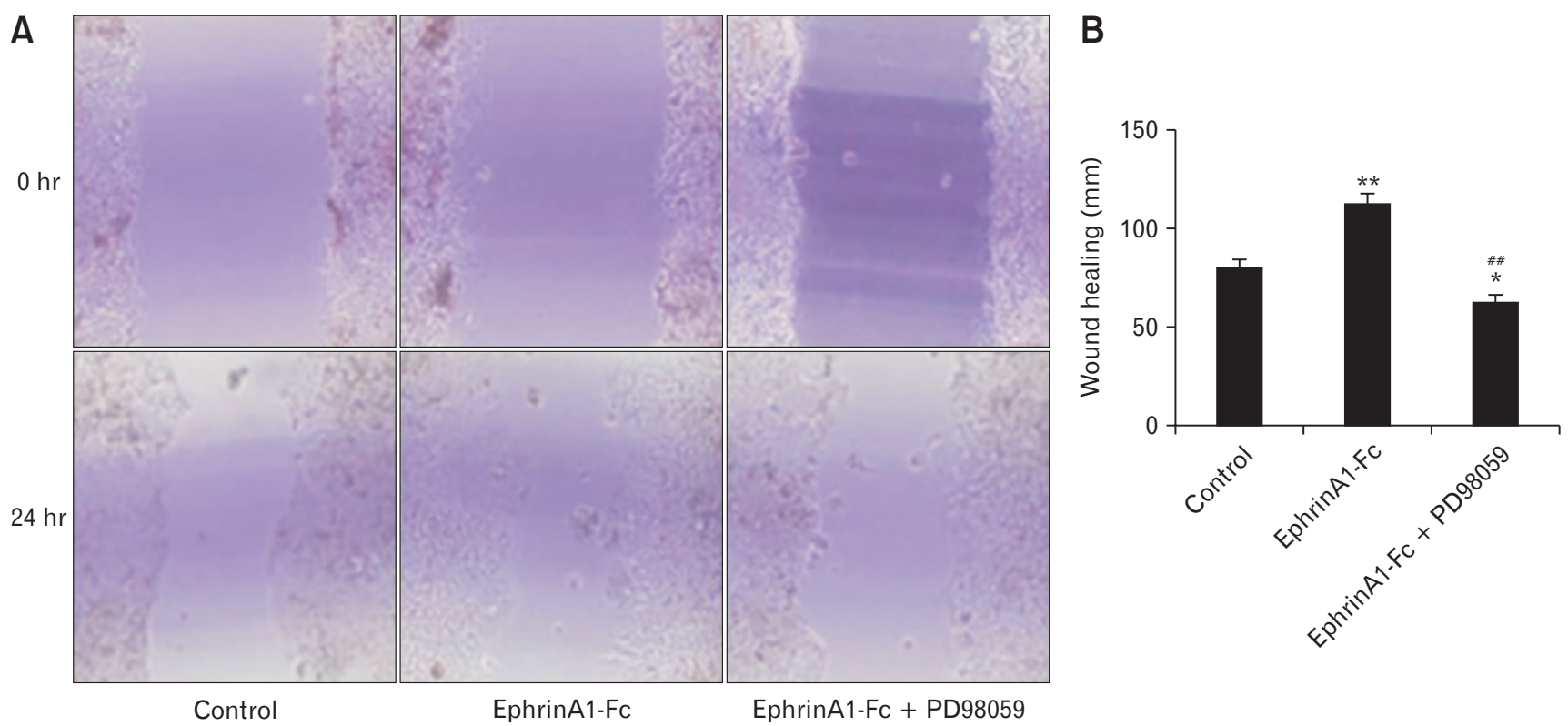

Figure 8. EphrinA1/ephA2 facilitated the cell migration of Caco2 cells. (A) Typical scratch wound assay of Caco2 monolayers at 0 hour and 24 hours. EphrinA1-Fc chimera (ephrinA1-Fc) accelerated the cell migration and wound repair, which indicated weakened intracellular tight junctions. Extracellular signal-regulated kinase 1/2 (ERK1/2) inhibition with ERK1/2 inhibitor (PD98059) prevented the cell migration mediated by ephrinA1-Fc. (B) Quantification of cell migration and wound healing. $\mathrm{n}=8,{ }^{*} P<0.05$, ${ }^{*} P<0.01$ vs controls; ${ }^{\# \#} P<0.01$ vs ephrinA1-Fc.

for regulating endotoxin-induced inflammatory cytokines in LPSinduced lung injury, including IL-1 $\beta$, IL-6, TNF- $\alpha$, and macrophage inflammatory protein $2 .^{15}$ They indicated that the increased ephrinA1/ephA2 activity was associated with LPS-mediated inflammatory state. In addition, LPS induced significant increase in the expression of ephrinA1, but not ephA2, in the Caco2 monolayer. Notably, the tyrosine phosphorylated level of ephA2 receptor was apparently evaluated in response to LPS. Therefore, it further confirmed the potential roles of ephrinA1/ephA2 in the disruptive effects of LPS.

EphrinA1/ephA2 signaling was associated with epithelial barrier disruption under conditions of LPS treatment. We found a negative correlation between ephrinA1/ephA2 levels and the epithelial permeability of FD4 and FD40 with decreased TEER and destroyed tight junctions. Recently, it reported that ephA2 play a central role in regulating the paracellular permeability of lung and brain endothelial cells, ${ }^{16,17}$ which may support our results observed in LPS-treated colon. More directly, we showed that specific ephA2 inactivation by ephA2-Fc and ephA2-mab obviously inhibited the hyperpermeability in LPS-exposed Caco2 monolayer, with increased TEER and decreased FD4 leakage. On the contrary, ephrinA1-Fc activated ephA2 receptors that mimicked the actions of LPS in barrier disruption. Overall, ephrinA1/ephA2 mediated the LPS-induced intestinal epithelial hyperpermeability, which may act as a potential interference target.

EphrinA1/ephA2 receptor has a critical role in modulating the intercellular junctions, such as adherent junction and tight junction, ${ }^{21}$ and mediate repulsive interaction between adjacent cells. ${ }^{20,22}$ Ephrin-A1 stimulation led to rearrangements of tight junctions and adherent junctions in bovine pulmonary artery endothelial cells. ${ }^{16}$ EphA2 receptor dismissed the expression of E-cadherin, which is involved in cell-to-cell adhesion, in microvascular endothelial cells. ${ }^{15,16}$ However, tight junctions are the main structures in determining the intestinal epithelial permeability. ${ }^{23,24}$ LPS induced apparent disruption of tight junctions and declined expression of integral membrane proteins such as occludin and claudin-1, the important tight junction proteins on the colonic columnar epithelium. ${ }^{25}$ Inhibition of ephrinA1/ephA2 augmented the expression of occludin and claudin-1, and improved the tight junctional structures that destroyed by LPS in Caco2 monolayer. Furthermore, from the perspective of clinical significance, for the scratch wound assay in this study, activated ephA2 can promote cell migration by disrupting intercellular tight junctions, therefore high intercellular permeability can exacerbate intestinal inflammation and even lead to systemic disease. ${ }^{26}$ EphA2 affects paracellular permeability of normal epithelial cells depending on its kinase activity, which promote disassembly of the tight junction proteins, such as claudin- $4,{ }^{21}$ occludin, and zonula occludens- $1 .{ }^{13}$ 
The ERK1/2 pathway is an essential player in many cellular and physiological functions, including proliferation, differentiation, and transcription. ${ }^{27,28}$ Tyrosine phosphorylated ephA2 promotes the nucleus translocation and p-ERK1/2 by forming a molecular complex with the Src homology and collagen (SHC) and growth factor receptor-bound protein 2 (GRB2) adaptor proteins. ${ }^{29} \mathrm{In}$ deed, ephrinA1-Fc stimulated apparent activation of ERK1/2 in Caco2 monolayers. Moreover, LPS led to significant p-ERK1/2 while blocked by ephA2 blocking with ephA2-Fc and ephA2-mab. It indicated that ephrinA1/ephA2-ERK1/2 cascade was activated in LPS exposed state. ERK1/2 has been confirmed to negatively regulate the tight junction functions, inhibiting the levels of claudin-1 and occludin in a variety of epithelial cells. ${ }^{30-33}$ The epithelial hyperpermeability and declined expression of occludin and claudin-1 induced by LPS, as well as ephrinA1-Fc, was improved by ERK1/2 inhibition with PD98059. Thereby, the ephrinA1/ephA2ERK1/2 signaling may be involved in the regulation of intestinal epithelial barrier function challenged with LPS.

In conclusion, ephrinA1/ephA2 kinase activation promotes epithelial hyperpermeability with an ERK1/2-dependent way, which is involved in LPS-induced intestinal barrier dysfunction. It is suggested that ephA2 receptor inhibitors may function as alternative therapeutic agents for LPS-induced intestinal barrier dysfunction. The identification of a role of the critical molecules in regulating barrier function can be a compelling strategy for effective and specific anti-inflammatory treatments.

Financial support: This study was supported by grants from the National Natural Science Foundation of China (NSFC), China (Grant No. 81570486, 81800463, and 81720108006).

\section{Conflicts of interest: None.}

Author contributions: Yongbo Zhang and Yuhua Chen performed the experiments, analyzed the data, and drafted the manuscript; Tao Bai and Wei Qian directed the completion of the cell model; Jun Song supported for the manuscript revision; and Xiaohua Hou and Lei Zhang designed and supervised the study, and obtained fundings.

\section{References}

1. Rosadini CV, Kagan JC. Early innate immune responses to bacterial LPS. Curr Opin Immunol 2017;44:14-19.

2. Hoogland IC, Houbolt C, van Westerloo DJ, van Gool WA, van de Beek D. Systemic inflammation and microglial activation: systematic re- view of animal experiments. J Neuroinflammation 2015;12:114.

3. Cavaillon JM. Exotoxins and endotoxins: inducers of inflammatory cytokines. Toxicon 2018;149:45-53.

4. Vespasiani-Gentilucci U, Gallo P, Picardi A. The role of intestinal microbiota in the pathogenesis of NAFLD: starting points for intervention. Arch Med Sci 2018;14:701-706.

5. Chen X, Zhao HX, Bai C, Zhou XY. Blockade of high-mobility group box 1 attenuates intestinal mucosal barrier dysfunction in experimental acute pancreatitis. Sci Rep 2017;7:6799.

6. Netto Candido TL, Bressan J, Alfenas RCG. Dysbiosis and metabolic endotoxemia induced by high-fat diet. Nutr Hosp 2018;35:1432-1440.

7. Dimitrijević M, Stanojević S, Vujić V, Aleksić I, Pilipović I, Leposavić G. Aging oppositely affects TNF-alpha and IL-10 production by macrophages from different rat strains. Biogerontology 2014;15:475-486.

8. Hayes CL, Dong J, Galipeau HJ, et al. Commensal microbiota induces colonic barrier structure and functions that contribute to homeostasis. Sci Rep 2018;8:14184.

9. Guo S, Al-Sadi R, Said HM, Ma TY. Lipopolysaccharide causes an increase in intestinal tight junction permeability in vitro and in vivo by inducing enterocyte membrane expression and localization of TLR-4 and CD14. Am J Pathol 2013;182:375-387.

10. Guo S, Nighot M, Al-Sadi R, Alhmoud T, Nighot P, Ma TY. Lipopolysaccharide regulation of intestinal tight junction permeability is mediated by TLR4 signal transduction pathway activation of FAK and MyD88. J Immunol 2015;195:4999-5010.

11. Tu J, Xu Y, Xu J, Ling Y, Cai Y. Chitosan nanoparticles reduce LPSinduced inflammatory reaction via inhibition of NF-kappaB pathway in Caco-2 cells. Int J Biol Macromol 2016;86:848-856.

12. Parri M, Buricchi F, Taddei ML, et al. EphrinA1 repulsive response is regulated by an EphA2 tyrosine phosphatase. J Biol Chem 2005;280:34008-34018.

13. Nasreen N, Khodayari N, Sriram PS, Patel J, Mohammed KA. Tobacco smoke induces epithelial barrier dysfunction via receptor EphA2 signaling. Am J Physiol Cell Physiol 2014;306:C1154-C1166.

14. Hong HN, Won YJ, Shim JH, et al. Cancer-associated fibroblasts promote gastric tumorigenesis through EphA2 activation in a ligandindependent manner. J Cancer Res Clin Oncol 2018;144:1649-1663.

15. Carpenter TC, Schroeder W, Stenmark KR, Schmidt EP. Eph-A2 promotes permeability and inflammatory responses to bleomycin-induced lung injury. Am J Respir Cell Mol Biol 2012;46:40-47.

16. Zhou N, Zhao WD, Liu DX, et al. Inactivation of EphA2 promotes tight junction formation and impairs angiogenesis in brain endothelial cells. Microvasc Res 2011;82:113-121.

17. Hong JY, Shin MH, Douglas IS, et al. Inhibition of EphA2/EphrinA1 signal attenuates lipopolysaccharide-induced lung injury. Clin Sci (Lond) 2016;130:1993-2003.

18. Xiong Y, Li KX, Wei H, Jiao L, Yu SY, Zeng L. Eph/ephrin signalling serves a bidirectional role in lipopolysaccharideinduced intestinal injury. Mol Med Rep 2018;18:2171-2181.

19. Rodiño-Janeiro BK, Alonso-Cotoner C, Pigrau M, Lobo B, Vicario M, Santos J. Role of corticotropin-releasing factor in gastrointestinal permeability. J Neurogastroenterol Motil 2015;21:33-50. 
20. Coulthard MG, Morgan M, Woodruff TM, et al. Eph/Ephrin signaling in injury and inflammation. Am J Pathol 2012;181:1493-1503.

21. Tanaka M, Kamata R, Sakai R. EphA2 phosphorylates the cytoplasmic tail of claudin- 4 and mediates paracellular permeability. J Biol Chem 2005;280:42375-42382.

22. Funk SD, Orr AW. Ephs and ephrins resurface in inflammation, immunity, and atherosclerosis. Pharmacol Res 2013;67:42-52.

23. Vancamelbeke $M$, Vermeire $S$. The intestinal barrier: a fundamental role in health and disease. Expert Rev Gastroenterol Hepatol 2017;11:821834.

24. Piche T. Tight junctions and IBS--the link between epithelial permeability, low-grade inflammation, and symptom generation? Neurogastroenterol Motil 2014;26:296-302.

25. Liang GH, Weber CR. Molecular aspects of tight junction barrier function. Curr Opin Pharmacol 2014;19:84-89.

26. Rodríguez-Fandiño OA, Hernández-Ruiz J, López-Vidal Y, CharúaGuindic L, Escobedo G, Schmulson MJ. Maturation phenotype of peripheral blood monocyte/macrophage after stimulation with lipopolysaccharides in irritable bowel syndrome. J Neurogastroenterol Motil 2017;23:281-288.

27. Aggarwal S, Suzuki T, Taylor WL, Bhargava A, Rao RK. Contrasting effects of ERK on tight junction integrity in differentiated and underdifferentiated Caco-2 cell monolayers. Biochem J 2011;433:51-63.

28. Hamaoka Y, Negishi M, Katoh H. EphA2 is a key effector of the MEK/ERK/RSK pathway regulating glioblastoma cell proliferation. Cell Signal 2016;28:937-945.

29. Pratt RL, Kinch MS. Activation of the EphA2 tyrosine kinase stimulates the MAP/ERK kinase signaling cascade. Oncogene 2002;21:76907699.

30. Perez White BE, Getsios S. Eph receptor and ephrin function in breast, gut, and skin epithelia. Cell Adh Migr 2014;8:327-338.

31. Guo H, Miao H, Gerber L, et al. Disruption of EphA2 receptor tyrosine kinase leads to increased susceptibility to carcinogenesis in mouse skin. Cancer Res 2006;66:7050-7058.

32. Baldwin C, Chen ZW, Bedirian A, et al. Upregulation of EphA2 during in vivo and in vitro renal ischemia-reperfusion injury: role of Src kinases. Am J Physiol Renal Physiol 2006;291:F960-F971.

33. Zhai Z, Ni X, Jin C, et al. Cecropin A modulates tight Junction-related protein expression and enhances the barrier function of porcine intestinal epithelial cells by suppressing the MEK/ERK pathway. Int J Mol Sci 2018;19:1941 